\title{
Singular length dependence of critical current in SNS bridges
}

\author{
Alex Levchenko, ${ }^{1}$ Alex Kamenev ${ }^{1}$ and Leonid Glazman ${ }^{1,2}$ \\ ${ }^{1}$ Department of Physics, University of Minnesota, Minneapolis, MN 55455, USA \\ ${ }^{2}$ W.I. Fine Theoretical Physics Institute, University of Minnesota, Minneapolis, MN 55455, USA
}

(Dated: October 25, 2006)

\begin{abstract}
We examine dependence of the critical Josephson current on the length $L$ of the normal bridge $\mathrm{N}$ between two bulk superconductors. This dependence turns out to be nonanalytic at small $L$. The nonanalyticity originates from the contribution of extended quasiparticle states with energies well above the superconducting gap. This should be contrasted with the more familiar contribution to the Josephson current coming from Andreev bound states localized in the normal region at energies below the gap.
\end{abstract}

PACS numbers: $74.50 .+\mathrm{r}, 74.45 .+\mathrm{c}$

Owing to the rapid progress in nanofabrication $1.2,3.4$, there is a renewed interest in various aspects of the physics of superconductor/normalmetal/superconductor (SNS) structures ${ }^{5}$. The purpose of this paper is to point out a subtle contribution to the Josephson current, flowing across the normal region. Specifically, we address the current carried by the high energy, $\varepsilon>\Delta$, extended states of the system. It leads to a nonanalytic behavior of the critical current as a function of the length of the normal region, $L$, at small $L$.

It is frequently stated 6.7 that as the result of Andreev reflection $\frac{13}{}$ the Josephson current is carried exclusively by the Andreev bound states with the energies $\varepsilon<\Delta$, localized in the normal region. Indeed, the energies of such bound states are sensitive to the phase difference, $\phi$, between the superconductors. This leads to the $\phi-$ dependent free energy and thus to the supercurrent. The validity of this point of view is well established in the two limiting cases of long9,10,11 junction $L \gg \xi$, and very short $\frac{6.7 .8}{.6}$ one, $L / \xi \rightarrow 0$, where $\xi$ is the coherence length of the superconductor. Thus it came as a surprise for us that in between these two extremes, the physics appears to be more complicated. We find that the Josephson current is shared between the localized Andreev states and the extended above-gap states. The dependence of the critical current on $L$ at short lengths, $L \ll \xi$, is not analytic and comes from the contribution of the extended states.

The phase sensitivity of the energy levels originates from the trajectories, which are reflected at least once from both NS interfaces. Such trajectories, require propagation time longer than the diffusion time across the normal region $L^{2} / D$, where $D$ is the diffusion constant. As a result, only states with energy smaller than the Thouless energy, $\varepsilon \lesssim E_{\mathrm{Th}}=\hbar D / L^{2}$, may exhibit sensitivity to the phase difference. In the long junction limit, the Thouless energy is small compared to the superconducting gap, $E_{\mathrm{Th}} \ll \Delta$ (indeed, notice that $\Delta=\hbar D / \xi^{2}$ ). Thus all the current-carrying states are at the energy $\varepsilon \lesssim E_{\mathrm{Th}} \ll \Delta$. Since there are no states in the superconducting leads at such energies, the Andreev bound states of the normal region are indeed solely responsible for the
Josephson current.

The situation is more complicated for shorter junctions, such that $E_{\mathrm{Th}}>\Delta$. One may expect that the extended states in the energy range $\Delta<\varepsilon<E_{\mathrm{Th}}$ contribute to the supercurrent. Yet, in the only case treated analytically so far, namely in the limit $L / \xi \rightarrow 0$ (or, equivalently, $E_{\mathrm{Th}} \rightarrow \infty$ ) the entire current is still given by the Andreev states $\frac{6.8}{6}$ with $0<\varepsilon \leq \Delta$. In this paper, we show that this result is an artefact of the limit $E_{\mathrm{Th}} \rightarrow \infty$ rather than a general principle. Specifically, the spectral density of the Josephson current appears to be $J(\varepsilon) \sim \Delta^{2} /\left(E_{\mathrm{Th}} \varepsilon\right)$ in the energy interval $\Delta \lesssim \varepsilon \lesssim E_{\text {Th }}$. Upon integration over energy, it results in the contribution to the critical current of the form $\delta I_{c} \sim\left(\Delta^{2} / E_{\mathrm{Th}}\right) \ln \left(\Delta / E_{\mathrm{Th}}\right)$, nonanalytic in the limit of the short normal region.

Summarizing the existing knowledge, one may write for the critical current of an SNS structure

$$
I_{c}=\frac{g \Delta}{e} \mathcal{I}(\kappa), \quad \kappa \equiv \frac{\Delta}{E_{\mathrm{Th}}},
$$

where $g$ is the conductance of normal region and $\mathcal{I}(\kappa)$ is a universal scaling function of the junction's dimensionless length $\kappa=(L / \xi)^{2}$. In the limit of long junction, $\kappa \gg 1$,

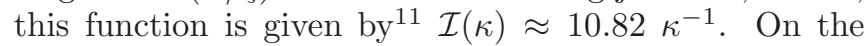
other hand, for shorter junctions, $\kappa \ll 1$, it is

$$
\mathcal{I}(\kappa)=\mathcal{I}(0)-a \kappa \ln (b / \kappa)+O\left(\kappa^{2}\right),
$$

where $\mathcal{I}(0)=2.08$, see, e. g., Ref. 6, and $a=0.31$, while $b=2.84$. The first term on the right-hand side (r.h.s.) represents the current carried by the Andreev states and was discussed by Kulik and Omelyanchuk ${ }^{8}$ and Beenakker $\underline{\underline{6}}$. The second nonanalytic term originates from the above-gap extended states. This term is the main result of the present paper. In the rest of the paper, we derive it and compare our analytical result with the existing $\frac{11}{11}$ numerical data.

To approach the problem analytically, we employ the Usadel equation $\frac{12}{2}$ for the matrix Green function $\hat{G}(\varepsilon, x)$ of the quasi-1D disordered normal region,

$$
D \partial_{x}\left(\hat{G} \partial_{x} \hat{G}\right)+\mathrm{i} \varepsilon\left[\hat{\sigma}_{3}, \hat{G}\right]=0,
$$


where the Green function is subject to the constraint $\hat{G}^{2}=\hat{1}$. At the two NS interfaces, the Green functions $\hat{G}(\varepsilon, \pm L / 2)$ are given by those of the BCS superconductors with the order parameter $\Delta$, maintained at the phase difference $\phi$. The suppression of the order parameter within the bulk superconducting leads may be safely disregarded as long as the transverse directions of the normal region are less than the coherence length, $\xi$. The same assumption justifies keeping only the lowest transverse mode and thus the use of the 1D form of the Usadel equation. This should be contrasted to the case of the long junction $L \gg \xi$, where fully self-consistent calculation is required and the approximation with the constant order parameter within the normal region is not appropriate.

The Green function may be parametrized by the two complex angles $\theta(\varepsilon, x)$ and $\varphi(\varepsilon, x)$ as

$$
\hat{G}=\sin \theta \cos \varphi \hat{\sigma}_{1}+\sin \theta \sin \varphi \hat{\sigma}_{2}+\cos \theta \hat{\sigma}_{3} .
$$

Employing this parametrization and introducing the rescaled coordinate $x / L \rightarrow x$, we rewrite the Usadel equation (33) as

$$
\partial_{x}^{2} \theta+\omega^{2} \sin \theta=J^{2} \cos \theta(\sin \theta)^{-3},
$$

where $\omega^{2} \equiv 2 \mathrm{i} \varepsilon / E_{\mathrm{Th}}$, and the spectral current density $J(\varepsilon)$ is given by

$$
J=\sin ^{2} \theta \partial_{x} \varphi .
$$

One needs to solve Eq. (5) with the boundary conditions

$$
\tan \theta(\varepsilon, \pm 1 / 2)=\mathrm{i} \Delta / \varepsilon .
$$

The spectral current density may then be determined from the condition of having the fixed phase difference: $\varphi(\varepsilon, 1 / 2)-\varphi(\varepsilon,-1 / 2)=\phi$. Finally, the Josephson current is found as

$$
I(\phi)=\frac{g}{e} \int_{0}^{\infty} \mathrm{d} \varepsilon \tanh \left(\frac{\varepsilon}{2 T}\right) \operatorname{Im} J(\varepsilon, \phi) .
$$

For the sake of illustration, we shall first execute this program in the short junction limit, $L \sim \omega \rightarrow 0$. Equation (5) with $\omega=0$ may be easily integrated, resulting in

$$
\cos \theta(\varepsilon, x)=\cos \theta_{0} \cos \left(\frac{J x}{\sin \theta_{0}}\right),
$$

where the integration constant is $\theta_{0}(\varepsilon)=\theta(\varepsilon, 0)$. Integrating then Eq. (6) and employing the boundary condition for the phase $\varphi$, one obtains

$$
\tan (\phi / 2)=\frac{1}{\sin \theta_{0}} \tan \left(\frac{J}{2 \sin \theta_{0}}\right) .
$$

This last equation along with Eq. (9) taken at the NS interface, $x=1 / 2$, constitute the system of the two algebraic equations for the two unknown quantities: $J(\varepsilon)$ and $\theta_{0}(\varepsilon)$. Such an algebraic problem may be easily solved, resulting in the following expression for the imaginary part of the spectral current:

$$
\operatorname{Im} J=\frac{\pi \Delta \cos (\phi / 2)}{\sqrt{\varepsilon^{2}-\Delta^{2} \cos ^{2}(\phi / 2)}}
$$

for $\Delta \cos (\phi / 2)<\varepsilon<\Delta$, and $\operatorname{Im} J=0$ otherwise. The fact that $\operatorname{Im} J$ vanishes for $\varepsilon>\Delta$ is an artefact of the approximation $E_{\mathrm{Th}} \rightarrow \infty$, already mentioned in the beginning of this paper. Equation (11) is in perfect agreement with the result of Beenakker $\underline{6}$ based on the consideration of the Andreev bound states in the diffusive normal region. One concludes that the Josephson current in the limit $L \rightarrow 0$ is entirely given by the Andreev states residing inside the superconducting gap. Employing Eqs. (8) and (11) one arrives at the well-known result $\underline{6.8}$ for the zero-temperature Josephson current of the short SNS junction,

$$
I(\phi)=I_{o} \cos (\phi / 2) \operatorname{arctanh}[\sin (\phi / 2)] .
$$

with $I_{o}=\pi g \Delta / e$. At $\phi_{\max }=1.97$, this function reaches its maximum value $I_{c} \equiv I\left(\phi_{\max }\right)=2.08 g \Delta / e$, which defines the coefficient $\mathcal{I}(0)=2.08$ in Eq. (2).

Having established the limit of an extremely short junction, we turn now to our main subject: the finitelength correction to the critical current. As was mentioned in the introductory section and will be proven below, the largest correction originates from the parametrically wide range of energies well above the superconducting gap: $\Delta<\varepsilon<E_{\mathrm{Th}}$. One may notice that in this energy interval, the solution for $\theta(\varepsilon)$ must be of the order of $\Delta / \varepsilon \ll 1$, allowing for small- $\theta$ expansion in Eq. (5),

$$
\partial_{x}^{2} \theta+\omega^{2} \theta=J^{2} \theta^{-3} .
$$

Within the same approximation, the boundary conditions (7) $\operatorname{read} \theta(\varepsilon, \pm 1 / 2)=\mathrm{i} \Delta / \varepsilon$, while Eq. (6) for the spectral current density takes the form $J=\theta^{2} \partial_{x} \varphi$.

Equation (13) may be solved exactly, leading to

$$
\theta^{2}(\varepsilon, x)=\theta_{0}^{2} \cos ^{2}(\omega x)+\frac{J^{2}}{\theta_{0}^{2}} \frac{\sin ^{2}(\omega x)}{\omega^{2}},
$$

where $\theta_{0}(\varepsilon)=\theta(\varepsilon, 0)$ is the integration constant, similar to the one in Eq. (9). Substituting the solution Eq. (14) into the equation for the spectral current and integrating over the coordinate, one finds

$$
\tan (\phi / 2)=\frac{J}{\theta_{0}^{2}} \frac{\tan (\omega / 2)}{\omega} .
$$

Notice that in the limit $\omega \rightarrow 0$ Eqs. (14) and (15) reduce to the small $\theta_{0}$ and small $J$ limit of Eqs. (9) and (10), respectively, as they should.

Taking Eq. (14) at the NS interface, $x=1 / 2$, and employing the boundary conditions, one obtains the algebraic relation between yet unknown quantities $\theta_{0}$ and 
$J$. The second relation between these two quantities is provided by Eq. (15). Resolving these two algebraic relations, one finds for the spectral current density

$$
J=-\frac{\Delta^{2}}{\varepsilon^{2}} \frac{\omega}{\sin \omega} \sin \phi .
$$

In the short junction limit, $\omega \rightarrow 0$, this expression is real, which is in agreement with our previous finding that $\operatorname{Im} J=0$ for $\varepsilon>\Delta$. However, for a finite-length junction (recalling that $\omega=\sqrt{2 \mathrm{i} \varepsilon / E_{\mathrm{Th}}}$ ) one finds the non-zero imaginary part of the spectral current: $\operatorname{Im} J \approx$ $-\Delta^{2} /\left(3 E_{\mathrm{Th}} \varepsilon\right)$ in the energy range $\Delta<\varepsilon<E_{\mathrm{Th}}$. For larger energies, $\varepsilon>E_{\mathrm{Th}}$, Eq. (16) predicts exponentially decaying $\operatorname{Im} J \propto \exp \left(-\sqrt{\varepsilon / E_{\mathrm{Th}}}\right)$. Indeed, as was discussed in the introductory part, the energies above the Thouless energy should not contribute to the supercurrent. Note that the tail of the spectral current found in the Eq. (16) at the energies above the gap is in perfect agreement with the results of the numercial studies $\frac{17}{}$. Integrating Eq. (16) over the energy according to Eq. (8), one finds the finite-size correction to the $T=0$ Josephson current, $\stackrel{14}{=}$

$$
\delta I(\phi)=-I_{o}\left(\frac{\kappa}{3 \pi}\right) \ln (b / \kappa) \sin \phi .
$$

This correction modifies the value of the critical current, $\delta I_{c}=\delta I\left(\phi_{\max }\right)$, with $\phi_{\max }$ quoted after Eq. (12). This way we obtain the second term in the r.h.s. of Eq.(2) with the coefficient $a=\left(\sin \phi_{\max }\right) / 3=0.31$.

We comment here on the additional approximation made while deriving Eq. (17). Our solution strategy was based on the perturbation theory for the Usadel equation over $L / \xi \ll 1$ exploring smallness on the phase $\theta(x, \varepsilon)$ in the energy range $\Delta<\varepsilon<E_{\mathrm{Th}}$. But we have used rigid boundary conditions (7) ignoring corrections to them coming from the finiteness of the normal region link. Although these corrections exist, they do not bring any new contributions non-analytical in $L / \xi$ to Eq. (17).

The crossover between the limits of short and long junctions was recently studied numerically by Dubos et

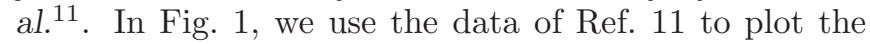
scaling function $\mathcal{I}(\kappa)$. At small $\kappa$, we expect the linear dependence of $[\mathcal{I}-\mathcal{I}(0)] / \kappa$ on $\ln \kappa$, see Eq. (2). The numerical data 11 indeed agree with this expectation, as shown in the inset to Fig. 1]

All the above consideration was based on the assumption that the interfaces between normal metal and superconductor are perfectly transparent. In most experimental configurations, SNS junctions always contain the potential barriers, an insulating layer I, at the SN interfaces. Thus it is of interest to investigate the influence of the extended quasiparticle energy levels on the Josephson current in the realistic SINIS configuration. In what follows, we will constraint ourselves to the case of symmetric SINIS junction with tunnel barrier conductance $g_{T}$ being much smaller then $g$.

The physics of the Josephson effect in the SINIS junction is controlled by the dimensionless interface parameter $\gamma_{\text {eff }}=(L / \xi)^{2}\left(g / g_{T}\right)$. In the so-called incoherent

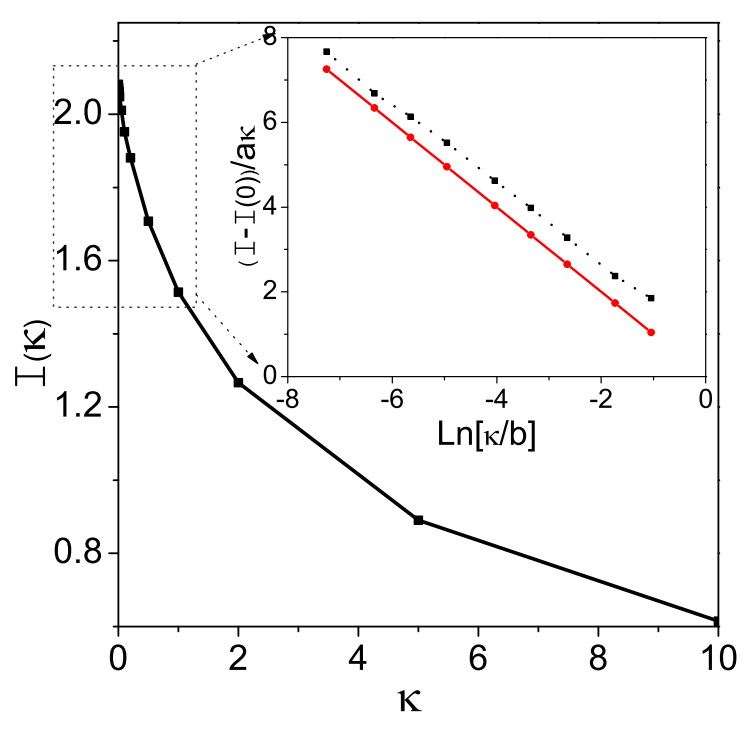

FIG. 1: (Color online) Numerical results of Ref ${ }^{11}$ for the scaling function $\mathcal{I}(\kappa)$. Inset: to facilitate the comparison of numerical results (Ref.11) (data points connected by a dashed line) with analytical theory [solid straight line corresponding to Eq. (2)], the data are replotted in co-ordinates $[\mathcal{I}-\mathcal{I}(0)] / a \kappa$ vs $\ln (\kappa / b)$ for the region $\kappa \leq 1$.

regime $\gamma_{\text {eff }} \gg 1$, the SINIS junction may be viewed as the two tunnel junctions connected in series with the current-phase relation in the form $I(\phi)=I_{c} \sin (\phi / 2)^{\frac{5}{5}}$. The most interesting case is the coherent regime $\gamma_{\text {eff }} \ll 1$ which we consider below.

To calculate the supercurrent for the SINIS junction, we start from the point contact limit and follow the same steps (5)-(10) as for SNS case but with one significant difference, namely the boundary conditions for Usadel equation. Instead of rigid boundary condition (7), we apply conditions appropriate for this case $\stackrel{15}{\longleftarrow}$

$$
J=\frac{2 g_{T}}{g} \sin \theta_{B} \sin \theta_{S} \sin \frac{\phi-\varphi_{B}}{2},
$$

$\left.\partial_{x} \theta\right|_{x=\frac{1}{2}}=\frac{2 g_{T}}{g}\left[\sin \theta_{S} \cos \theta_{B} \cos \frac{\phi-\varphi_{B}}{2}-\sin \theta_{B} \cos \theta_{S}\right]$,

where $\theta_{B}(\varepsilon)=\theta(\varepsilon, \pm 1 / 2), \varphi_{B}=\varphi(\varepsilon, 1 / 2)$ and $\cos \theta_{S}=$ $\varepsilon / \sqrt{\varepsilon^{2}-\Delta^{2}}$. As long as the tunnel conductance is small $g_{T} \ll g$ the phase function $\varphi(\varepsilon, x)$ changes abruptly at the tunnel barriers from its values $\pm \phi / 2$ inside the superconductors to $\pm \varphi_{B}$ at the boundaries of the junction and remains small inside the normal region $|\varphi(\varepsilon, x)| \ll 1$. These observations implies that in the boundary conditions (18) one can safely put $\varphi_{B}=0$. As follows from the Eq.(18) the spectral current $J$ is small as $\sim g_{T} / g \ll 1$. Therefore, according to the solution (9) of the Usadel equation the $\theta(\varepsilon, x)$ is essentially coordinate independent 
$\partial_{x} \theta(x, \varepsilon)=0$, provided $E_{\mathrm{Th}} \gg \Delta g / g_{T}$. In this case the boundary conditions (18) represent the closed system of algebraic equations for unknown functions $\theta_{B}$ and $J$, which can be solved in terms of $\phi$ and $\theta_{S}$. As a result the imaginary part of the spectral current takes the form

$$
\operatorname{Im} J=\frac{g_{T}}{g} \frac{\Delta^{2} \sin \phi}{\sqrt{\Delta^{2}-\varepsilon^{2}} \sqrt{\varepsilon^{2}-\Delta^{2} \cos ^{2}(\phi / 2)}} .
$$

Combining this with Eq. (8) at zero temperature, we find the Josephson current-phase relation in the form $\frac{15}{16}$

$$
I(\phi)=I_{o}\left(2 g_{T} / \pi g\right) K[\sin (\phi / 2)] \sin \phi,
$$

where $K(x)$ is the complete elliptic integral of the first kind. Let us compare this result with Eq.(12). First of all, we observe that the presence of the tunnel barriers changes the current-phase relation, but preserves its essential properties, for example nonanalyticity at phase $\phi=\pi$. Secondly, the amplitude of the critical current is suppressed by the small parameter $g_{T} / g \ll 1$ compared to its value, Eq.(12). Similar changes occur with the Josephson current correction $\delta I$ : we get modified phase$\phi$ dependence, the amplitude of the current correction is suppressed due to the tunnel barriers, but the correction itself remains nonanalytical in $L / \xi$. The estimate shows that the amplitude of the Josephson current correction in the symmetric SINIS junction is

$$
\delta I_{c} \propto-I_{o}\left(g_{T} / g\right) \kappa \ln (1 / \kappa) .
$$

Let us discuss now some characteristic features of the above-gap contribution to the Josephson current found in this paper: (i) The power-law tail of the spectral current density, $\operatorname{Im} J \sim 1 / \varepsilon$, results in the nonanalytic length dependence of the critical current, $\delta I_{c} \sim L^{2} \ln L$. (ii) The supercurrent carried by the high-energy extended states is negative, i.e., it flows in the direction opposite to the current produced by the Andreev bound states. (iii) Unlike the dependence on length, the phase dependence of the found contribution to Josephson current is not singular, $\delta I(\phi) \sim \sin \phi$. This should be contrasted with the contribution coming from the Andreev bound states, Eq. (12). The latter contains (at any $L$ ) a nonanalytic phase dependence, $I(\phi) \sim(\pi-\phi) \ln |\pi-\phi|$, at $\phi \approx \pi$. The origin of such a difference between the two contributions is in the evolution of the Andreev states with the phase $\phi$ : unlike above-gap states, the lowest of the bound states depends on the phase in a peculiar way, "touching" the Fermi level, $\varepsilon=0$, at $\phi=\pi$. (iv) The occupation of the states contributing to the $L^{2} \ln L$ dependence of the Josephson current hardly changes with temperature, as long as $T \lesssim \Delta(T)$. As a result, the temperature dependence of the second term in the r.h.s. of Eq. (2) comes solely from the temperature-dependent superconducting gap $\Delta(T)$. (v) At a temperature close to the critical one: $T_{c}-T \ll T_{c}<E_{\mathrm{Th}}$, the short SNS bridge is in the regime where $\Delta(T) \ll T$. Performing energy integration according to Eq. (8), one finds for the Josephson current:

$$
I(\phi)+\delta I(\phi)=\frac{g \Delta^{2}(T)}{e}\left(\frac{\pi}{4 T}-\frac{\ln \left(E_{\mathrm{Th}} / T_{c}\right)}{3 E_{\mathrm{Th}}}\right) \sin \phi,
$$

where the first term in brackets originates from the Andreev states, while the second one originates from the high energy extended states. The nonanalytic length dependence exists in this case as well.

Although our calculations were carried out for the case of the diffusive normal region, it is clear that the effect is rather generic. In particular, with the proper redefinition of the Thouless energy (and possibly with different numerical constants), Eqs. (11) and (2) should hold for ballistic SNS structures as well18. Moreover, nonanalytical correction survives even in the case of SINIS junction but with suppressed amplitude due to small tunnel barriers conductance. Also the critical current of superconducting weak links of the type SS'S should contain the nonanalytic term, originating from the high-energy states, and numerical studies $\frac{17}{}$ only support this conclusion.

We are grateful to H. Courtois, F.K. Wilhelm and Levy Yeyati for useful discussions, and H. Courtois for providing us with the results of their numerical calculation 11 , which enabled us to perform the comparison presented in Fig. 1. This work is supported by NSF grants DMR 0237296, DMR 04-39026 DMR 0405212, and EIA 02-10736. A. K. is also supported by the A.P. Sloan foundation.
1 Y. Doh, J.A. Dam, A.L. Roest, E. Bakkers, L.P. Kouwenhoven, S. Franceschi, Science 309, 272, (2005).

2 A. Kasumov, M. Kociak, M. Ferrier, R. Deblock, S. Gueron, B. Reulet, I. Khodos, O. Stephan, H. Bouchiat, Phys. Rev. B 68, 214521, (2003).

3 B.J. van Wees, H. Takayanagi, Proceeding of the NATO Advanced Study Institute on Mesoscopic Electron Transport, edited by L.P.Kouwenhoven, p.469, (1996).

4 B.J. Wees, Physics World, 9(11), 41, (1996).

5 A.A. Golubev, M.Yu. Kupriyanov, E.Il'ichev, Rev. Mod. Phys. 76, 411, (2004).
${ }^{6}$ C.W.J. Beenakker, Phys. Rev. Lett. 67, 3836, (1991) and Transport Phenomena in Mesoscopic Systems, edited by H. Fukuyama and T. Ando (Springer, Berlin, 1992).

7 T.T. Heikkila, J. Sarkka and F.K. Wilhelm, Phys. Rev. B 66, 184513, (2002).

8 I.O. Kulik, A.N. Omelyanchuk, Sov. J. Low. Temp. Phys, 3, 459, (1977) and 4, 142, (1978).

9 F.K. Wilhelm, A.D. Zaikin, G.Schon, J. Low Temp. Phys. 106, 305, (1997).

${ }^{10}$ W. Belzig, F.K. Wilhelm, C. Bruder, G. Shön, A.D. Zaikin, Superlattices Microstruct. 25, 1251, (1999). 
11 P. Dubos, H. Courtois, B. Pannetier, F.K. Wilhelm, A.D. Zaikin and G. Schön, Phys. Rev. B 63, 064502, (2001).

12 K.D. Usadel, Phys. Rev. Lett, 25, (1970).

13 A.F. Andreev, Sov. Phys. JETP, 19, 1228, (1964).

14 Finding the coefficient $b=2.84$ inside the logarithm requires more careful consideration of the energy range $\varepsilon \approx \Delta$, where $\theta$ is not small and one has to work with Eq. (5), rather than Eq. (13); A. Levchenko, A. Kamenev, L. Glazman, unpublished.
15 M.Yu. Kupriyanov, V.F. Lukichev, Sov. Phys. JETP, 67, 1163, (1988)

16 A. Brinkman, A.A. Golubov, Phys. Rev. B 61, 11297, (2000)

17 A. Levy Yeyati, A. Martn-Rodero, and F. J. Garca-Vidal, Phys. Rev. B, 51, 3743 (1995)

18 S.V. Sharov, A.D. Zaikin, Phys. Rev. B 71, 014518, (2005) 